\title{
Transparent Conductive Oxides for the Epsilon-Near-Zero Tamm Plasmon Polaritons
}

\author{
Rashid G. Bikbaev1,2, , Stepan Ya. Vetrov ${ }^{2,1}$, and Ivan V. Timofeev ${ }^{1,2}$ \\ ${ }^{1}$ Kirensky Institute of Physics, Federal Research Center KSC SB RAS, Krasnoyarsk 660036, Russia \\ ${ }^{2}$ Siberian Federal University, Krasnoyarsk 660041, Russia \\ *Corresponding author: bikbaev@iph-krasn.ru
}

Compiled September 17, 2019

\begin{abstract}
We demonstrated the possibility of using transparent conducting oxides (AZO, GZO, ITO) to form Tamm plasmon polaritons in the near-infrared spectral range where the permitivity of oxides is near-zero. The spectral properties of the structures have been investigated in the framework of the temporal coupled mode theory and confirmed by transfer matrix method. It was found that in the critical coupling conditions the maximal Q-factor of a Tamm plasmon polariton is achieved when photonic crystal is conjugated with the AZO film, while at the conjugation with the ITO films the broadest spectral line is obtained. The sensitivity of the wavelength and spectral width of the Tamm plasmon polariton to changes of the oxide film thickness, the bulk concentration of a dopand and the angle of incidence are demonstrated. (๑) 2019 Optical Society of America
\end{abstract}

http://dx.doi.org/10.1364/ao.XX.XXXXXX

\section{INTRODUCTION}

In recent years, much attention has been paid to the Tamm plasmon polariton (TPP), which represents a standing surface wave that does not transfer energy [1]. Such a wave can be formed at the interface between two reflecting media, e.g., a photonic crystal (PC) and a planar metallic film in case of normal incidence of light on the structure. In this case, the field amplitude at the TPP wavelength is localized at the interface between media and exponentially decreases on both sides. In the energy spectra of a sample, the TPP can be seen as a narrow peak [2-4]. The spectral properties of the TPP can be controlled by varying the lattice parameters [5] or metallic film material [6]. The TPPs gave rise to the fundamentally new type of devices, which includes absorbers [7-10], switches [11], organic solar cells [12], thermal emitters [13, 14], sensors [15, 16] and spontaneous emission amplifiers [17]. In addition to metal, the resonant nanocomposite materials can be used as a material with negative dielectric permittivity for TPP excitation [18-22]. Moreover, by these materials TPPs can be formed when the real part of the permittivity is near-zero (ENZ) in visible wavelength range. In this case, unlike conventional TPP, the localized state is realized in the spectral region in which the real part of dielectric permittivity is several orders of magnitude smaller than its imaginary part. Thus the reflection of the ENZ material is largely determined by the imaginary part of the dielectric permittivity [23]. Therefore, the use of such materials broadens the understanding of TPP and the environment for its implementation. Another example of materials with near-zero permittivity in addition to nanocomposite are transparent conducting oxides (TCO), e.g. ITO (Indium tin oxide), AZO (Aluminium doped zinc oxide), and GZO (Gallium doped zinc oxide).

These oxides are an alternative to the classical plasmon materials, such as silver and gold [24-29]. They are attractive due to the possibility of implementing the high nonlinearity [3032], designing modulators [33] and polarization devices [34], bending the wave front [35] and creating absorbers [36] and insulators [37]. All this was obtained due to the unique dispersion properties of the TCOs in the epsilon-near-zero wavelength range. In this work, we show the possibility of using these materials to form TPPs and control their spectral properties. It should be noted that the use of TCO allows to obtain localized states in the near-infrared spectral range where permittivity of the oxides is near-zero. Therefore, the results are new in comparison with the works already performed on this topic $[38,39]$ The formation of ENZ TPP in this spectral range makes it promising to use the proposed model for using it as photonics elements with tunable optics properties.

\section{MODEL DESCRIPTION}

We consider a PC structure that represents a layered medium bound by a finite TCO layer (Fig. 1).

The PC unit cell is formed from materials $a$ and $b$ with layer thicknesses $d_{a}$ and $d_{b}$ and permittivities $\varepsilon_{a}$ and $\varepsilon_{b}$. The PC structure is here in after assumed to be placed in vacuum.

The permittivity of TCOs in the wavelength range from 0.3 to $2 \mu \mathrm{m}$ can be described by the Drude-Lorentz model [24]: 


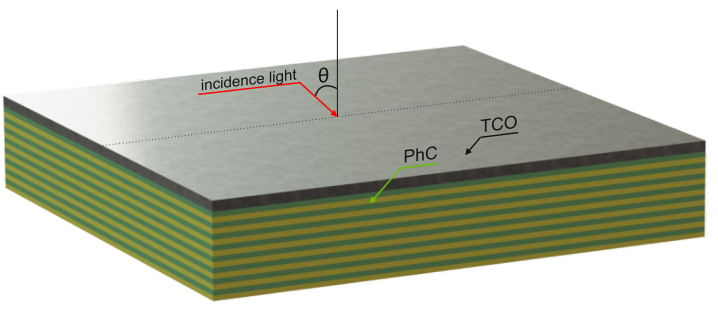

Fig. 1. Schematic representation of $\mathrm{PhC}$ bounded by TCO layer.

$$
\varepsilon(\omega)=\varepsilon_{0}-\frac{\omega_{p}^{2}}{\omega(\omega+i \gamma)}+\frac{f_{1} \omega_{1}^{2}}{\omega_{1}^{2}-\omega\left(\omega+i \gamma_{1}\right)} .
$$

The Drude parameters for different TCOs materials are shown in table 1.

Table 1. Drude parameters for TCOs materials

\begin{tabular}{cccccccc}
\hline TCO & $\varepsilon_{0}$ & $\omega_{p}(\mathrm{eV})$ & $\gamma(\mathrm{eV})$ & $f_{1}(\mathrm{eV})$ & $\omega_{1}(\mathrm{eV})$ & $\gamma_{1}(\mathrm{eV})$ & $\lambda_{\text {ENZ }}(\mathrm{nm})$ \\
\hline AZO & 3.5402 & 1.7473 & 0.04486 & 0.5095 & 4.2942 & 0.1017 & 1416 \\
GZO & 3.2257 & 1.9895 & 0.1229 & 0.3859 & 4.05 & 0.0925 & 1184 \\
ITO & 3.528 & 1.78 & 0.1549 & 0.3884 & 4.21 & 0.0919 & 1389 \\
\hline
\end{tabular}

Figure 2 shows the dependence of the TCO permittivity on the incident light wavelength calculated using Eq. 1.

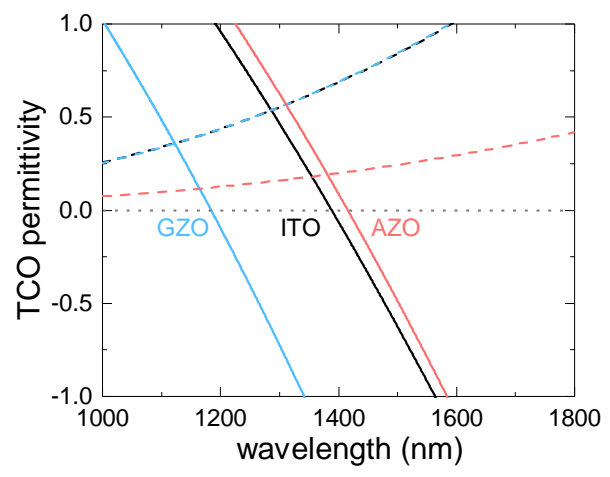

Fig. 2. Dependences of the real (solid line) and imaginary (dashed line) parts of the TCO permittivity on the incident radiation wavelength.

It can be seen that the real part of the permittivity takes nearzero value in the near-infrared (IR) spectral range. In particular, we have $\Re \varepsilon_{A Z O} \approx 0$ at $\lambda=1416 \mathrm{~nm}, \Re \varepsilon_{G Z O} \approx 0$ at $\lambda=1184 \mathrm{~nm}$ and $\Re \varepsilon_{\text {ITO }} \approx 0$ at $\lambda=1389 \mathrm{~nm}$ (see. Table 1 ). We will seek for localized states in the vicinity of these points.

\section{COUPLED MODE THEORY}

According to temporal coupled mode theory [40, 41], any state (resonance) has its own frequency $\omega_{0}$ and number $N$ of energy flow outside and inside the resonance. In this case, the energy loss in the channels is described by the relaxation times $\tau_{l}$, where $l=1 \ldots N$. We assume that the state is described by complex amplitude $A$ and energy $|A|^{2}$.

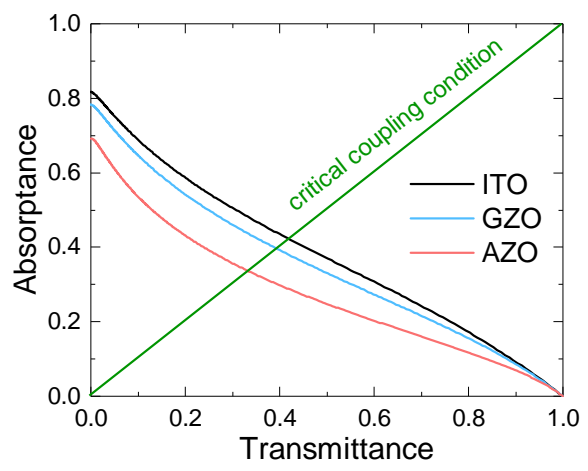

Fig. 3. Dependence of the absorptance of the TCO films on their transmittance at different film thicknesses. The points of intersection of the lines with the green line meet critical coupling conditions (Eq. 13).

The state can be presented as a sum of the incoming and outgoing energy flows with amplitudes $s_{l \pm}$. If the energy leaves the state along two energy channels with relaxation times $\tau_{1}$ and $\tau_{2}$, then the relaxation time of the state is determined as $1 / \tau=1 / \tau_{1}+1 / \tau_{2}$. In this case, the variation in amplitude $A$ obeys the equation:

$$
d A / d t=-i \omega_{0} A-A / \tau,
$$

which has the solution $A(t)=A(0) e^{-i \omega_{0} t-t / \tau}$.

We can rewrite Eq. 2 with regard to the amplitudes of the incoming and outgoing energy flows $s_{l \pm}$ as:

$$
d A / d t=-i \omega_{0} A-A / \tau_{1}-A / \tau_{2}+\alpha_{1} s_{1+}+\alpha_{2} s_{2+},
$$

where $s_{l-}=\beta_{l} s_{l-}+\gamma_{l} A$. Here $\alpha_{l}$ and $\gamma_{l}$ are the quantities that characterize the channel coupling and $\beta$ is the coefficient of energy reflection in the channel. The model, however, can adequately describe the properties of the localized state even with disregard of these parameters. It is sufficient to know the relaxation time $\tau$ and frequency $\omega_{0}$ of the state. The final form of the change in the amplitude of the localized state field is

$$
\frac{d A}{d t}=-i \omega_{0} A-\sum_{l=1}^{2} A / \tau_{l}+\sum_{l=1}^{2} \sqrt{\frac{2}{\tau_{l}}} s_{l+},
$$

and the amplitudes of the flows are related as:

$$
s_{l-}=-s_{l+}+\sqrt{\frac{2}{\tau_{l}}} A .
$$

Using Eq. 4 and Eq. 5 , we may predict the transmission spectrum of the structure. In the general case, the transmittance is a ratio between the amplitudes of the incident and transmitted waves $T(\omega)=\left|s_{2-}\right|^{2} /\left|s_{1+}\right|^{2}$, at $s_{2+}=0$. The incident radiation with frequency $\omega$ will decay according to the law $e^{-i \omega t}$, and $d A / d t=-i \omega A$. Under these conditions, Eq. 4 and Eq. 5 can be written in the form

$$
\begin{aligned}
& -i \omega A=-i \omega_{0} A-\sum_{l=1}^{2} A / \tau_{l}+\sqrt{\frac{2}{\tau_{1}}} s_{1+}, \\
& s_{1-}=-s_{1+}+\sqrt{\frac{2}{\tau_{1}} A,} s_{2-}=\sqrt{\frac{2}{\tau_{2}}} A
\end{aligned}
$$


a)
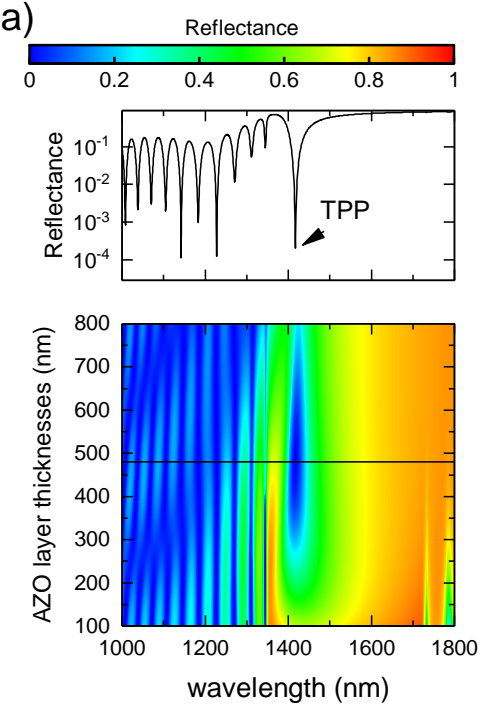

b)
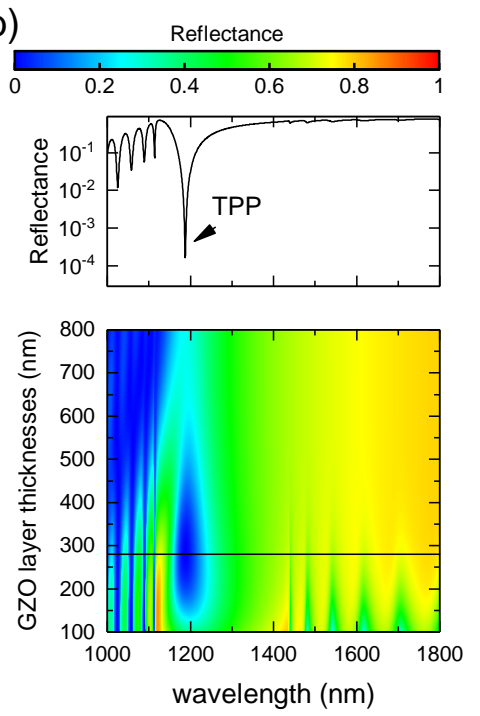

c)
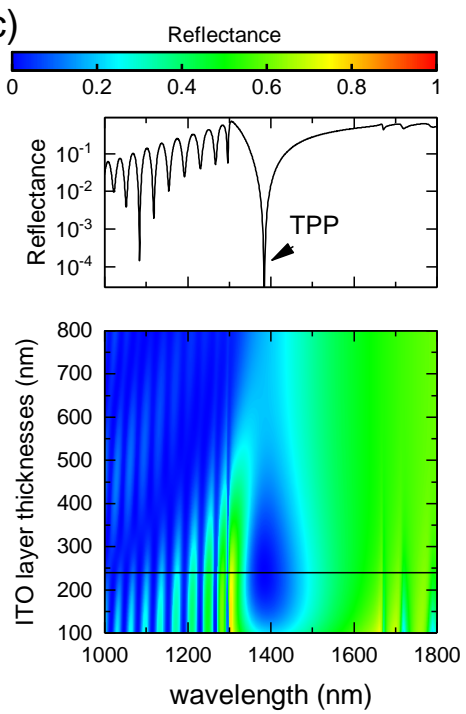

Fig. 4. Reflectance spectra of a PC conjugated with (a) AZO, (b) GZO and (c) ITO layers of different thicknesses.

and the transmittance as:

$$
T(\omega)=\frac{\frac{2}{\tau_{2}}|A|^{2}}{\left|s_{1+}\right|^{2}}=\frac{\frac{4}{\tau_{1} \tau_{2}}}{\left(\omega-\omega_{0}\right)^{2}+\left(\frac{1}{\tau_{1}}+\frac{1}{\tau_{2}}\right)^{2}} .
$$

The reflectance is determined analogously: :

$$
R(\omega)=\frac{\left|s_{1-}\right|^{2}}{\left|s_{1+}\right|^{2}}=\frac{\left(\omega-\omega_{0}\right)^{2}+\left(\frac{1}{\tau_{1}}-\frac{1}{\tau_{2}}\right)^{2}}{\left(\omega-\omega_{0}\right)^{2}+\left(\frac{1}{\tau_{1}}+\frac{1}{\tau_{2}}\right)^{2}} .
$$

The absorptance includes all the types of losses is determined from the energy conservation law $T(\omega)+R(\omega)+A(\omega)=1$.

These energy coefficients have the corresponding peaks in the form of Lorentzian contours with the full width at half maximum

$$
2 \gamma=\sum_{l=1}^{N} \frac{1}{\tau_{l}},
$$

where $\gamma$ is the amplitude relaxation rate, which is equal to the ratio between the power of energy relaxation to the channel and the energy accumulated in the state (resonance).

For one channel, we have $l=l_{1}$. In this case, the reflection amplitude can be written in the form

$$
r_{l}=-1+\frac{2 \gamma_{l}}{i\left(\omega_{0}-\omega\right)+\sum \gamma_{l_{1}}} .
$$

The zero reflection is only possible under the critical coupling conditions, i. e., at $\omega=\omega_{0}$ :

$$
r_{l}\left(\omega=\omega_{0}\right)=0 .
$$

\section{RESULTS AND DISCUSSION}

In the structure under study, three energy channels contribute to the TPP formation (Fig. 1).

We denote the rates of energy relaxation to the TCO transmission, absorption and PC transmission channel by $\gamma_{T C O}, \gamma_{A}$ and $\gamma_{P h C}$, respectively. The state relaxation rate is the ratio between the leakage power and the accumulated energy. In particular, for the radiation incident onto and reflected from the TCO layer (first channel), the relaxation rate is $\gamma_{T C O}$. For the radiation absorbed by the TCO film (the second channel), it is $\gamma_{A}$. The radiation incident from the side of the Bragg mirror makes the third energy channel, which is characterized by the relaxation rate $\gamma_{P h C}$.

Since the energy accumulated in the TPP is the same for determining the rate of relaxation to each channel, the relaxation rates and corresponding energy coefficients of the structure are related as [14]:
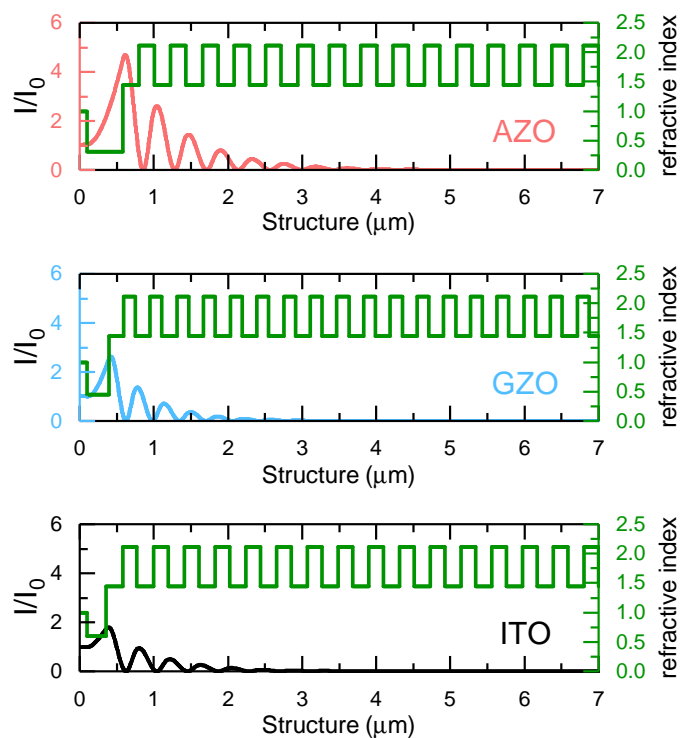

Fig. 5. Local field intensity distribution at the TPP wavelength normalized to the input intensity for the cases of conjugation of a PC with the AZO, GZO and ITO films. The green line shows the spatial distribution of the refractive index of the PC layers. The simulation parameters are presented in table 2 .

$$
\gamma_{T C O}: \gamma_{A}: \gamma_{P h C}=T_{T C O}: A_{T C O}: T_{P h C}
$$



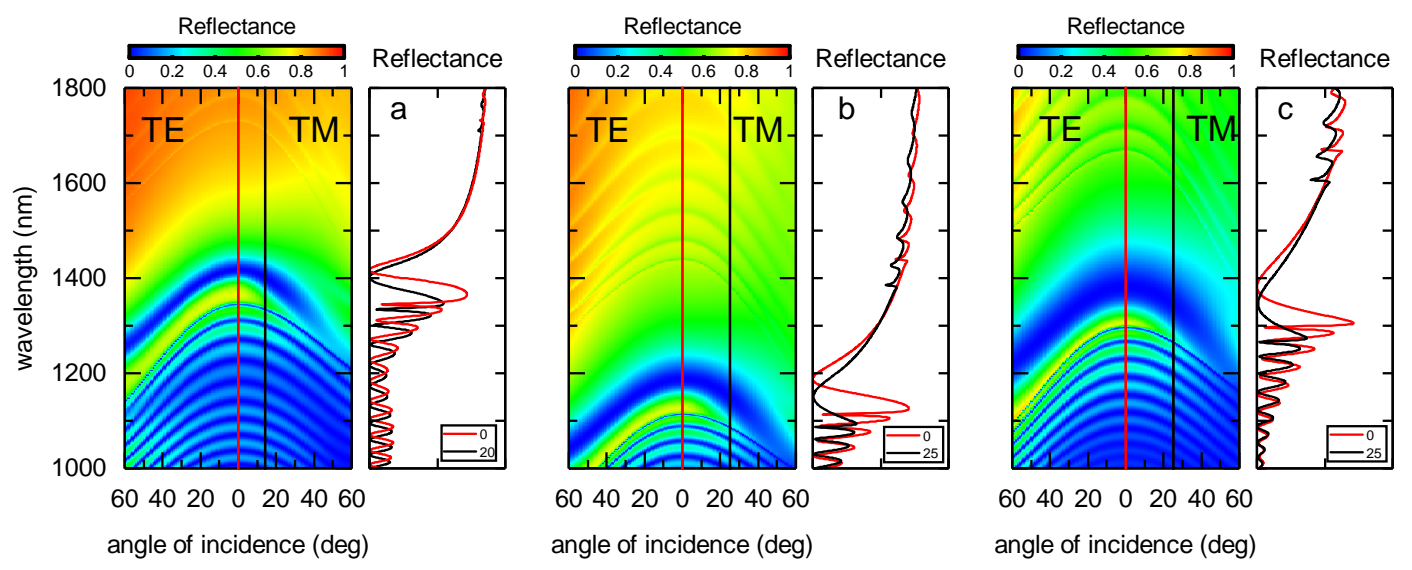

Fig. 6. Reflectance spectra for (a) the AZO-PhC, (b) GZO-PhC and (c) ITO-PhC structures at different angles of incidence of the radiation onto the structure.

Under excitation of the TPP from the TCO and opaque PC side, one of the energy relaxation channels can be ignored. Then, critical coupling condition (12) can be written in the form:

$$
\gamma_{T C O}=\gamma_{A} ; \quad \gamma_{P h C}=0 \Leftrightarrow T_{T C O}=A_{T C O} ; \quad T_{P h C}=0 .
$$

Critical coupling equation 13 for the AZO, GZO and ITO films can be solved graphically. To do this, we should build the dependence of the absorptance of the TCO films on their transmittance $A_{T C O}\left(T_{T C O}\right)$. We consider the TCO film with refractive index $n_{2}$, which is located between two dielectric media with the refractive indices $n_{1}=n_{3}=1$. The transmittance, reflectance and absorptance of the TCO film are determined using the Airy formulas

$$
\begin{gathered}
T_{T C O}=\frac{n_{3}}{n_{1}}\left|\frac{t_{12}+t_{23} e^{i \beta}}{1+r_{12} r_{23} e^{2 i \beta}}\right|^{2}, \quad R_{T C O}=\left|\frac{r_{12}+r_{23} e^{2 i \beta}}{1+r_{12} r_{23} e^{2 i \beta}}\right|^{2}, \\
A_{T C O}=1-T_{T C O}-R_{T C O}
\end{gathered}
$$

where $\beta=2 \pi n_{2} d_{T C O} / \lambda_{0}$ is the phase incoming during the passage of the layer by the wave; $\lambda_{0}$ is the wavelength; $d_{T C O}$ is the TCO film thickness; $t_{12}=2 n_{1} /\left(n_{1}+n_{2}\right), r_{12}=\left(n_{1}-\right.$ $\left.n_{2}\right) /\left(n_{1}+n_{2}\right)$ and $t_{23}=2 n_{2} /\left(n_{2}+n_{3}\right), r_{23}=\left(n_{2}-n_{3}\right) /\left(n_{2}+\right.$ $\left.n_{3}\right)$ - are the amplitudes of transmission and reflection at the interfaces 1-2 and 2-3.

The obtained dependences are presented in Fig. 3, which shows that for the AZO film the critical coupling condition is established at the lower transmittance and absorptance, i. e., at the lower energy relaxation rates the sum of which determines the resonance spectral linewidth. Thus, under the critical coupling conditions, the resonance line and, consequently, the absorption band of the AZO film are narrower than for the GZO and ITO films. In addition, it means that at the conjugation of a PC with the AZO film, the TPP Q factor is maximal.

To check this fact, we calculated the transmission spectra of the PC structure by the transfer matrix method [42], in which the amplitudes of the incident and outgoing waves are connected by the $2 \times 2$ matrices multiplication:

$$
M=T_{01} T_{12} \ldots T_{N-1, N} T_{N, S},
$$

where the transfer matrix is

$$
T_{n-1, n}=\frac{1}{2}\left(\begin{array}{cc}
(1+h) e^{-i \alpha_{n} \gamma_{n}} & (1-h) e^{i \alpha_{n} \gamma_{n}} \\
(1-h) e^{-i \alpha_{n} \gamma_{n}} & (1+h) e^{i \alpha_{n} \gamma_{n}}
\end{array}\right),
$$

Here $\left.h=\sqrt{\varepsilon_{n} / \varepsilon_{n-1}}, \varepsilon(n)\right)$ is the permittivity of the $n$th layer, $\alpha_{n}=(\omega / c) \sqrt{\varepsilon(n)}, c$ is the speed of light, $\gamma_{n}=\mathbf{z}_{n}-\mathbf{z}_{n-1}$ are the layer thicknesses $(n=1,2, \ldots, N), z_{n}$ is the coordinate of the interface between the $n$th layer and the $(n+1)$ th layer adjacent from the right, and $\gamma_{N+1}=0$. The transfer matrix for the orthogonally polarized wave is obtained from (16) by replacing $h$ for $\sqrt{\varepsilon_{n-1} / \varepsilon_{n}}$. The energy transmittance, reflectance, and absorbance are determined as

$T(\omega)=\frac{1}{\left|\hat{M}_{11}\right|^{2}}, \quad R(\omega)=\frac{\left|\hat{M}_{21}\right|^{2}}{\left|\hat{M}_{11}\right|^{2}}, \quad A(\omega)=1-T(\omega)-R(\omega)$.

where $\hat{M}_{11}, \hat{M}_{21}$ - are the elements of matrix $\hat{M}$.

For certainty, we take silicon dioxide $\left(\mathrm{SiO}_{2}\right)$ and zirconium dioxide $\left(\mathrm{ZrO}_{2}\right)$ as materials of the alternating PC layers. The number of PhC layers is $N=41$ for all the presented structures. The thicknesses of the PC layers conjugated with the AZO, GZO and ITO films are given in Table 2.

Table 2. Parameters of the structures

\begin{tabular}{cccccc}
\hline Structure & $d_{a}(\mathrm{~nm})$ & $d_{b}(\mathrm{~nm})$ & $d_{T C O}(\mathrm{~nm})$ & $\lambda_{T P P}(\mathrm{~nm})$ & $\varepsilon_{T C O}\left(\lambda_{T P P}\right)$ \\
\hline AZO-PhC & 220 & 208 & 480 & 1417 & $-0.011+0.2063 i$ \\
GZO-PhC & 200 & 160 & 280 & 1188 & $-0.024+0.424 i$ \\
ITO-PhC & 220 & 195 & 240 & 1381 & $0.034+0.6615 i$ \\
\hline
\end{tabular}

The calculated data are presented in Fig. 4. It can be seen that, inside the PC band gap, the TPPs is formed in the wavelength ranges where the real part of the TCO permittivity is near-zero. In particular, at the conjugation of a PC with the AZO film, the TPP wavelength is $\lambda=1417 \mathrm{~nm}$ and $\mathrm{Q} \approx 20$; at the conjugation with the GZO film $\lambda=1188 \mathrm{~nm}$ and $\mathrm{Q} \approx 11$; at the conjugation with the ITO film $\lambda=1381 \mathrm{~nm}$ and $\mathrm{Q} \approx 7$, respectively. The permittivities of the oxides at these wavelengths are $\varepsilon_{A Z O}=$ $-0.011+0.2063 i, \varepsilon_{G Z O}=-0.024+0.424 i$ and $\varepsilon_{I T O}=0.034+$ $0.6615 i$ (see. Table 2).

It is worth noting that the critical coupling condition is established at film thicknesses of $d_{A Z O}=480 \mathrm{~nm}, d_{G Z O}=280 \mathrm{~nm}$, $d_{\text {ITO }}=240 \mathrm{~nm}$ (Table 2). The TCO thickness in this case is much greater than thickness of metal film used for the formation of conventional TPP. A larger reflection from the TCO film with a small dielectric permittivity can be achieved by increasing its thickness. Therefore, it is preferable to use thick films of TCO to 


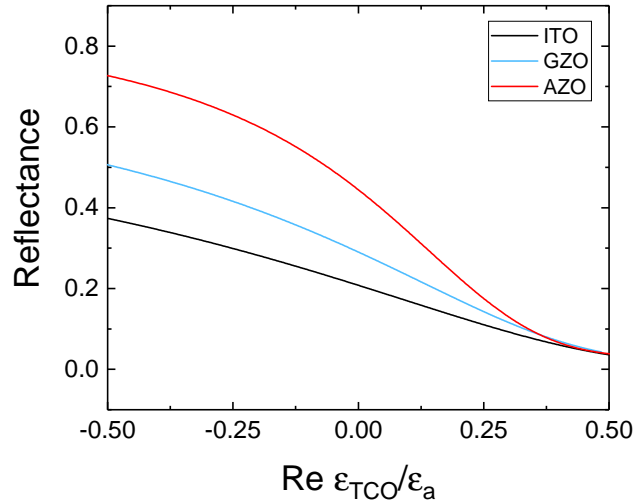

Fig. 7. Reflectance spectra of the air-TCO-silicon dioxide structures.

achieve the critical coupling condition of the incident field with TPP. Figure 4 shows that the spectral linewidth corresponding to the TPP is minimum at the conjugation of a PC with the AZO film $(Q \approx 20)$, which is consistent with the predictions of the temporal coupled mode theory. The TPP linewidth is maximal at the conjugation of the PC with the ITO film $(Q \approx 7)$. In this case, the broadband TPP is formed inside the PC band gap $[43,44]$.

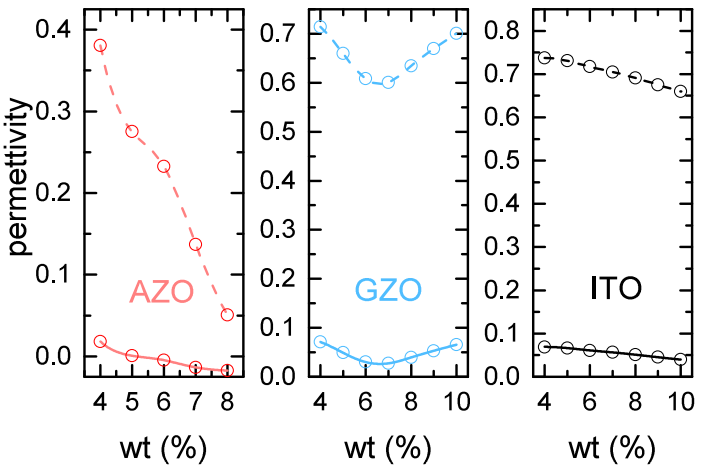

Fig. 8. Dependences of the real (solid line) and imaginary (dashed line) parts of the TCO permittivity on volume concentrations of the doping metal. The circles represent experimental data from [24] and solid lines represent interpolated data.

It should be noted that the TPP is formed not only for the negative real part of the permittivity of the TCO, but also for the positive ones (see. Table 2). We propose the following explanation of the TPP formation at the positive TCO permittivity. Figure 7 shows the calculated dependence of the reflection on $\operatorname{Re} \varepsilon_{T C O} / \varepsilon_{a}$ for the normal incidence of light on a finite TCO layer, which is located between $\mathrm{SiO}_{2}$ and air. Reflectances of the TCO films are determined using the Airy formulas (Eq. 14). It can be seen that at small positive and small negative $\operatorname{Re} \varepsilon_{T C O} / \varepsilon_{a}$ values, the reflection of the AZO, GZO and ITO attains $48 \%$, $29 \%$ and $20 \%$, respectively, which facilitates the formation of a localized state at the PhC-TCO interface in all three cases.

The spatial distributions of the local field intensity at the TPP frequencies are presented in Fig. 5. It can be seen that the light field localization at the TPP wavelength for the AZO-PhC
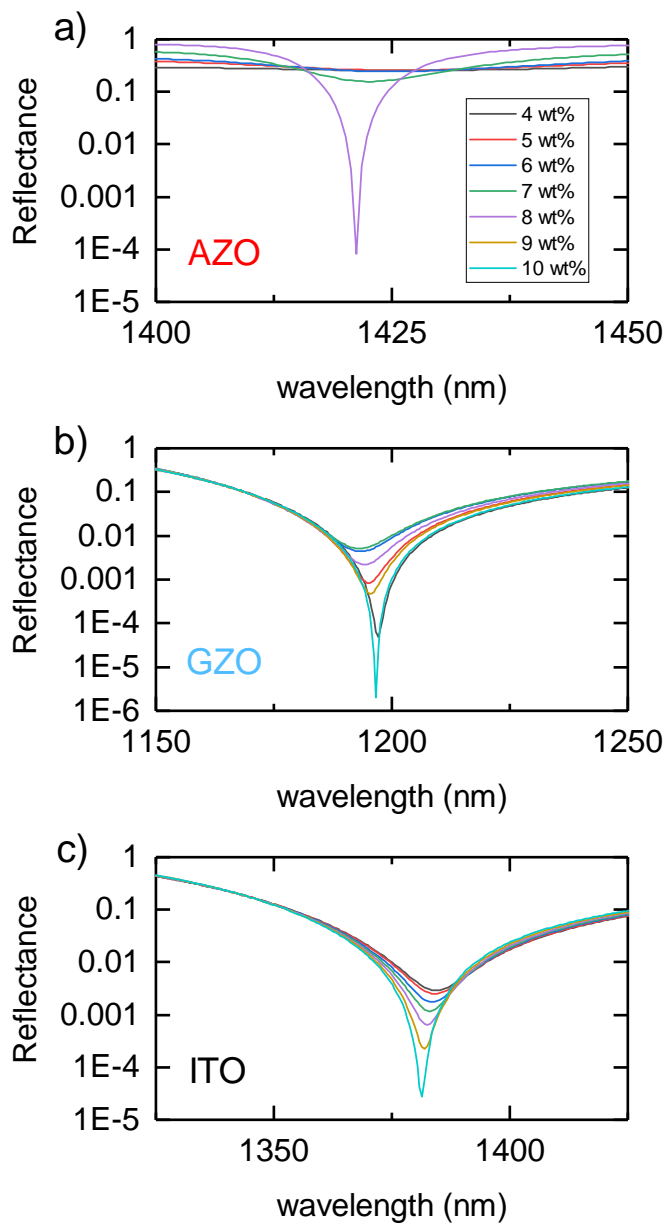

Fig. 9. Reflectance spectra for (a) the AZO-PhC, (b) GZO-PhC, and (c) ITO-PhC structures at different volume concentrations of the doping metal. The TCO layer thicknesses are $d_{A Z O}=$ $480 \mathrm{~nm}, d_{G Z O}=280 \mathrm{~nm}$ and $d_{I T O}=260 \mathrm{~nm}$. The legend is valid for all the three plots.

structure is higher by 77 and 152\% than for the GZO-PhC and ITO-PhC structures, respectively. In addition, note that the light field in the TPP is localized in the region comparable with the wavelength.

The change of the angle of incidence of the radiation onto the structure is an effective tool for controlling the TPP wavelength. Figure 6 shows the reflectance spectra of the investigated structures at different angles of incidence $\theta$ of the radiation.

It can be seen that for all the three investigated configurations, the TPP wavelength shifts to the shorter-wavelength spectral range for both the TE and TM modes. In this case, the TPP spectral linewidth increases only for the TM modes, while for the TE modes it remains nearly invariable. In order to estimate the spectral line broadening, we present (Fig. 6) the comparative transmittance spectra at $\theta=0^{\circ}$ for all the structures, $\theta=20^{\circ}$ for $\mathrm{AZO}-\mathrm{PhC}, \theta=25^{\circ}$ for GZO-PhC and ITO-PhC. In this case, the lines are broaden by 20,25 and $30 \%$, respectively. It should be noted that, here, the TPP is formed at the small positive values of the real part of the TCO permittivity. In particular, for the $\mathrm{AZO}-\mathrm{PhC}$ structure, at an angle of incidence of $\theta=20^{\circ}$, the TPP wavelength is $1386 \mathrm{~nm}$ and the permittivity takes the value $\varepsilon_{A Z O}=0.1645+0.1932 i$. The reflectance of the AZO 
film at these parameters is $25 \%$. This value is sufficient for TPP localization. In the GZO-PhC and ITO-PhC structures, the mechanism of the TPP formation is analogous to that described above.

The change of the volume concentration of a doping metal can be used to effectively control the optical properties of the TCO materials and as a results the TPP wavelength. In [24] authors have shown that the change of the volume concentration of a doping material leads to a significant change the $\gamma$ in Eq. 1 The dependencies of the dielectric permittivity of TCO on the volume concentration dopant metal are shown in figure 8 . The results are obtained at a fixed wavelength of incident radiation equal to the wavelength of TPP (see Table 2). It can be seen from this figure that in the studied range of volume concentrations the dielectric permittivity of $\mathrm{AZO}$ changes to a greater extent than for GZO and ITO. Thus, the reflection spectra of the AZO-PhC structure should be most sensitive to changes in the volume concentration of the dopant material. To verify this assumption, the reflectance spectra of the structure were calculated. The results are shown in figure 9. In Fig. 9b the change in the $G a$ volume concentration in zinc oxide leads to the blue shift of the TPP wavelength. For ITO, the picture is different, since the TPP wavelength undergoes the red shift (Fig. 9c). The maximal sensitivity of the reflectance at the TPP wavelength is attained at the conjugation of a PC with the AZO layer (Fig. 9a). In this case, a decrease in the $A l$ volume concentration from 8 to $7 \%$ and lower leads to a decrease in the reflectance at the localized state wavelength by more than three orders of magnitude. This behavior of the reflection coefficient at the wavelength of TPP is explained by the strong dependence of the optical properties of the AZO film on the volume concentration of $A l$. So the change in the concentration of $A l$ by $1 \mathrm{wt} \%$ (from 8 to 7 ) increases $\gamma$ nearly twice (from 0.0167 to 0.02963 ). As a result, the drastic variation of film dielectric constant leads to violation of the critical coupling conditions (Eq. 13). It should be noted that for GZO and ITO films the corresponding change in $\gamma$ is less than $7 \%$, and the detuning from the critical coupling conditions is slight.

\section{CONCLUSION}

We examined the TPPs localized at the interface between a PC and epsilon-near-zero TCO film. The possibility of the localized state formation was predicted analytically and implemented numerically by transfer-matrix method. Using the temporal coupled-mode theory, we have determined that for AZO-PhC structure TPP has a high Q factor while for ITO-PhC a spectral line is broader. These results were confirmed by the calculation. It was installed that the light field localization at the TPP wavelength for the AZO-PhC structure is higher by $77 \%$ and $152 \%$ than for the GZO-PhC and ITO-PhC structures, respectively. We established that the change in the volume concentration of the dopant makes it possible to effectively control the reflection at the TPP wavelength. In particular, for AZO film the change in the indium concentration by $1 \%$ (from 8 to $9 \%$ ) leads to the change in the reflectance at the TPP wavelength by more than three orders of magnitude. It was shown that the change in the angle of incidence of the radiation onto the structure is an effective way of controlling the TPP spectral linewidth and position inside the band gap. For the GZO and ITO films, the broadest spectral line is obtained at an angle of incidence of $25^{\circ}$. Our investigations develop the idea about the possible TPP formation at the interface between a PC and epsilon-near-zero materials.

\section{ACKNOWLEDGMENT}

The reported study was funded by RFBR according to the research projects № 18-32-00053. I.V.T. and S.Ya.V acknowledges financial support RFBR and MOST according to the research project № 19-52-52006.

\section{REFERENCES}

1. M. A. Kaliteevski, I. lorsh, S. Brand, R. A. Abram, J. M. Chamberlain, A. V. Kavokin, and I. A. Shelykh, "Tamm plasmon-polaritons: Possible electromagnetic states at the interface of a metal and a dielectric Bragg mirror," Phys. Rev. B 76, 165415 (2007).

2. M. E. Sasin, R. P. Seisyan, M. A. Kaliteevski, S. Brand, R. A. Abram, J. M. Chamberlain, A. Y. Egorov, A. P. Vasil'ev, V. S. Mikhrin, and A. V. Kavokin, "Tamm plasmon polaritons: Slow and spatially compact light," Appl. Phys. Lett. 92, 251112 (2008).

3. M. E. Sasin, R. P. Seisyan, M. A. Kaliteevski, S. Brand, R. A. Abram, J. M. Chamberlain, I. V. Iorsh, I. A. Shelykh, A. Y. Egorov, A. P. Vasil'ev, V. S. Mikhrin, and A. V. Kavokin, "Tamm plasmon-polaritons: First experimental observation," Superlattices Microstruct. 47, 44-49 (2010).

4. T. Goto, A. V. Dorofeenko, A. M. Merzlikin, A. V. Baryshev, A. P. Vinogradov, M. Inoue, A. A. Lisyansky, and A. B. Granovsky, "Optical tamm states in one-dimensional magnetophotonic structures," Phys. Rev. Lett. 101, 14-16 (2008).

5. X.-L. Zhang, J. Feng, X.-C. Han, Y.-F. Liu, Q.-D. Chen, J.-F. Song, and H.-B. Sun, "Hybrid Tamm plasmon-polariton/microcavity modes for white top-emitting organic light-emitting devices," Optica. 2, 579 (2015).

6. Che-Yuan Chang, Yi-Hsun Chen, Yu-Lin Tsai, Hao-Chung Kuo, and Kuo-Ping Chen, "Tunability and Optimization of Coupling Efficiency in Tamm Plasmon Modes," IEEE J. Sel. Top. Quantum Electron. 21, 262-267 (2015).

7. Y. Gong, X. Liu, L. Wang, H. Lu, and G. Wang, "Multiple responses of TPP-assisted near-perfect absorption in metal/Fibonacci quasiperiodic photonic crystal," Opt. Express 19, 9759 (2011).

8. Y. Gong, X. Liu, H. Lu, L. Wang, and G. Wang, "Perfect absorber supported by optical Tamm states in plasmonic waveguide," Opt. Express 19, 18393 (2011).

9. M. Fang, F. Shi, and Y. Chen, "Unidirectional All-Optical Absorption Switch Based on Optical Tamm State in Nonlinear Plasmonic Waveguide," Plasmonics 11, 197-203 (2016).

10. C.-H. Xue, F. Wu, H.-T. Jiang, Y. Li, Y.-W. Zhang, and H. Chen, "Wideangle Spectrally Selective Perfect Absorber by Utilizing Dispersionless Tamm Plasmon Polaritons," Sci. Reports 6, 39418 (2016).

11. W. Zhang and S. Yu, "Bistable switching using an optical Tamm cavity with a Kerr medium," Opt. Commun. 283, 2622-2626 (2010).

12. X.-L. Zhang, J.-F. Song, X.-B. Li, J. Feng, and H.-B. Sun, "Optical Tamm states enhanced broad-band absorption of organic solar cells," Appl. Phys. Lett. 101, 243901 (2012).

13. Z.-y. Yang, S. Ishii, T. Yokoyama, T. D. Dao, M.-g. Sun, T. Nagao, and K.-p. Chen, "Tamm plasmon selective thermal emitters," Opt. Lett. 41, $4453(2016)$

14. Z.-Y. Yang, S. Ishii, T. Yokoyama, T. D. Dao, M.-G. Sun, P. S. Pankin, I. Timofeev, T. Nagao, and K.-p. Chen, "Narrowband Wavelength Selective Thermal Emitters by Confined Tamm Plasmon Polaritons," ACS Photonics 4, 2212-2219 (2017).

15. S.-G. Huang, K.-P. Chen, and S.-C. Jeng, "Phase sensitive sensor on Tamm plasmon devices," Opt. Mater. Express 7, 1267 (2017).

16. B. Auguié, M. C. Fuertes, P. C. Angelomé, N. L. Abdala, G. J. A. A. Soler Illia, and A. Fainstein, "Tamm Plasmon Resonance in Mesoporous Multilayers: Toward a Sensing Application," ACS Photonics 1, 775-780 (2014).

17. A. R. Gubaydullin, C. Symonds, J. Bellessa, K. A. Ivanov, E. D. Kolykhalova, M. E. Sasin, A. Lemaitre, P. Senellart, G. Pozina, and M. A. Kaliteevski, "Enhancement of spontaneous emission in Tamm plasmon structures," Sci. Reports 7, 9014 (2017).

18. S. Y. Vetrov, R. G. Bikbaev, and I. Timofeev, "Optical Tamm states at 
the interface between a photonic crystal and a nanocomposite with resonance dispersion," J. Exp. Theor. Phys. 117, 988-998 (2013).

19. S. Y. Vetrov, R. G. Bikbaev, and I. Timofeev, "The optical Tamm states at the edges of a photonic crystal bounded by one or two layers of a strongly anisotropic nanocomposite," Opt. Commun. 395, 275-281 (2017).

20. S. Y. Vetrov, P. S. Pankin, and I. Timofeev, "The optical Tamm states at the interface between a photonic crystal and a nanocomposite containing core-shell particles," J. Opt. 18, 065106 (2016).

21. R. G. Bikbaev, S. Y. Vetrov, and I. Timofeev, "The optical Tamm states at the interface between a photonic crystal and nanoporous silver," J. Opt. 19, 015104 (2017).

22. R. G. Bikbaev, S. Y. Vetrov, and I. Timofeev, "Optical Tamm states at the interface between a photonic crystal and a gyroid layer," J. Opt. Soc. Am. B 34, 2198 (2017).

23. S. Y. Vetrov, R. G. Bikbaev, N. V. Rudakova, K.-p. Chen, and I. Timofeev, "Optical Tamm states at the interface between a photonic crystal and an epsilon-near-zero nanocomposite," J. Opt. 19, 085103 (2017).

24. G. V. Naik, J. Kim, and A. Boltasseva, "Oxides and nitrides as alternative plasmonic materials in the optical range [invited]," Opt. Mater. Express 1, 1090 (2011).

25. M. A. Noginov, L. Gu, J. Livenere, G. Zhu, A. K. Pradhan, R. Mundle, M. Bahoura, Y. A. Barnakov, and V. A. Podolskiy, "Transparent conductive oxides: Plasmonic materials for telecom wavelengths," Appl. Phys. Lett. 99, 021101 (2011).

26. G. V. Naik, V. M. Shalaev, and A. Boltasseva, "Alternative plasmonic materials: Beyond gold and silver," Adv. Mater. 25, 3264-3294 (2013).

27. G. Naik, J. Kim, N. Kinsey, and A. Boltasseva, "Alternative plasmonic materials," in Modern Plasmonics, (Elsevier, 2014), pp. 189-221.

28. J. Kim, G. V. Naik, N. K. Emani, U. Guler, and A. Boltasseva, "Plasmonic resonances in nanostructured transparent conducting oxide films," IEEE J. Sel. Top. Quantum Electron. 19, 4601907-4601907 (2013).

29. S. M. Choudhury, D. Wang, K. Chaudhuri, C. DeVault, A. V. Kildishev, A. Boltasseva, and V. M. Shalaev, "Material platforms for optical metasurfaces," Nanophotonics. 7, 959-987 (2018).

30. M. Z. Alam, I. De Leon, and R. W. Boyd, "Large optical nonlinearity of indium tin oxide in its epsilon-near-zero region," Science. 352, 795-797 (2016).

31. A. Ciattoni, C. Rizza, A. Marini, A. Di Falco, D. Faccio, and M. Scalora, "Enhanced nonlinear effects in pulse propagation through epsilon-nearzero media," Laser Photonics Rev. 10, 517-525 (2016).

32. R. P. M. Kaipurath, M. Pietrzyk, L. Caspani, T. Roger, M. Clerici, C. Rizza, A. Ciattoni, A. Di Falco, and D. Faccio, "Optically induced metal-to-dielectric transition in Epsilon-Near-Zero metamaterials," Nat. Publ. Group pp. 1-7 (2016).

33. I. C. Reines, M. G. Wood, T. S. Luk, D. K. Serkland, and S. Campione, "Compact epsilon-near-zero silicon photonic phase modulators," Opt. Express 26, 21594-21605 (2018).

34. Y. Xu and J. Xiao, "Design and numerical study of a compact, broadband and low-loss te-pass polarizer using transparent conducting oxides," Opt. Express 24, 15373-15382 (2016).

35. A. Alù, M. G. Silveirinha, A. Salandrino, and N. Engheta, "Epsilonnear-zero metamaterials and electromagnetic sources: Tailoring the radiation phase pattern," Phys. Rev. B 75, 155410 (2007).

36. J. Park, J.-H. Kang, X. Liu, and M. L. Brongersma, "Electrically Tunable Epsilon-Near-Zero (ENZ) Metafilm Absorbers," Sci. Reports 5, 15754 (2015).

37. A. Davoyan, A. Mahmoud, and N. Engheta, "Optical isolation with epsilon-near-zero metamaterials," Opt. Express 21, 3279-3286 (2013).

38. R. Bikbaev, S. Vetrov, and I. Timofeev, "Two types of localized states in a photonic crystal bounded by an epsilon near zero nanocomposite," Photonics 5, 22 (2018).

39. R. Bikbaev, S. Vetrov, and I. Timofeev, "Epsilon-near-zero absorber by tamm plasmon polariton," Photonics 6, 28 (2019).

40. H. Haus, Waves and fields in optoelectronics (Prentice-Hall, Englewood Cliffs, NJ, 1984).

41. J. D. Joannopoulos, S. G. Johnson, J. N. Winn, and R. D. Meade,
Photonic crystals : molding the flow of light (Princeton University Press, Princeton, 2008).

42. P. Yeh, "Electromagnetic propagation in birefringent layered media," J. Opt. Soc. Am. 69, 742 (1979).

43. S. E. Svyakhovskiy, R. G. Bikbaev, S. A. Myslivets, S. A. Evlashin, A. M. Vyunishev, P. S. Pankin, I. V. Timofeev, S. Y. Vetrov, and V. G. Arkhipkin, "Experimental demonstration of broadband optical tamm states in photonic crystal," in 2018 International Conference Laser Optics (ICLO), (2018), pp. 309-309.

44. A. M. Vyunishev, R. G. Bikbaev, S. E. Svyakhovskiy, I. V. Timofeev, P. S. Pankin, S. A. Evlashin, S. Y. Vetrov, S. A. Myslivets, and V. G. Arkhipkin, "Broadband tamm plasmon polariton," J. Opt. Soc. Am. B 36, 2299-2305 (2019). 